\title{
Seeing clearly through COVID-19: current and future questions for the history and philosophy of the life sciences
}

\author{
Giovanni Boniolo $^{1}$ (D) $\cdot$ Lisa Onaga $^{2}$ (D)
}

Accepted: 26 May 2021 / Published online: 14 June 2021

(C) Springer Nature Switzerland AG 2021

\begin{abstract}
The role of a journal like HPLS during the novel coronavirus pandemic should serve as a means for scholars in different fields and professions to consider historically and critically what is happening as it unfolds. Surely it cannot tackle all the possible issues related to the pandemic, in particular to the COVID-19 pandemic, but it does have a responsibility to foster the best possible dialogue about the various issues related to the history and philosophy of the life sciences, and thus to solicit contributions from potential authors working in different parts of the world and belonging to different cultural traditions. Only a real plurality of perspectives should allow for a better, large-scale comprehension of what the COVID-19 pandemic is.
\end{abstract}

Keywords COVID-19 - Global pandemic and the life sciences $\cdot$ Historical and philosophical studies of the novel coronavirus

The role of a journal like HPLS during the novel coronavirus pandemic should serve as a space in which scholars of different fields and professions are encouraged to consider historically and critically issues and events soon after they unfold. Surely it cannot tackle all the possible issues related to the pandemic, in particular to the COVID-19 pandemic, but it does have a responsibility to foster the best possible

Giovanni Boniolo

bnlgnn@unife.it

$\triangle$ Lisa Onaga

lonaga@mpiwg-berlin.mpg.de

1 Dipartimento di Neuroscienze e Riabilitazione, Università di Ferrara, Via Fossato di Mortara 64/A, 44121 Ferrara, Italy

2 Max Planck Institute for the History of Science, Boltzmann Str. 22, 14195 Berlin, Germany 
dialogue about the various issues related to the history and philosophy of the life sciences, and thus to solicit contributions from potential authors working in different parts of the world and belonging to different cultural traditions. Only a real plurality of perspectives should allow for a better, large-scale comprehension of what the COVID-19 pandemic is.

The essays appearing in the Topical Collection initiative "Seeing Clearly Through COVID-19" follow a short-form essay format. This departure from the traditional research article length papers that appear in HPLS Topical Collections reflects two key rationales. First of all, a short note could offer a sort of icastic reflection on an aspect of the pandemic, so that the potential reader could have synthetic but intriguing material to think about. These essays reflected a common prompt to deliver a clear, substantially justified-but open-perspective. Second, we thought that this short note format was an appropriate method with which to initially address the precarity that the COVID-19 pandemic has laid bare. At the time, this epidemic of global proportions had surprised many people, from laymen, laywomen and children, to politicians, economists, clinicians, biomedical researchers, and humanists.

The global pandemic has drastically changed our ways of living world-wide and will likely continue to change how we live in the future. Under these circumstances, historians of medicine of different traditions have importantly had to show that there have been precedents for the conditions leading up to and representing the disastrous effects taking place. The significance of the history of life sciences has never been more relevant. Analogously, philosophers of the life sciences of different traditions have had to critically analyse the situation. The combined historical and philosophical inquiry has the potential to design a framework that would permit a means to better understand the unusual and terrible events by practicing rigorous questiondriven analysis on the one hand, and by maintaining a historically empirical basis for reflection on the other hand. Thus, in order to pursue this two-part approach we reason why we hoped to receive submissions from all countries of world and from scholars coming from different scholarly traditions when we launched this Topical Collections. And, to our delight, we received an overwhelming response, which results in the forum you read now.

The over thirty papers curated in this collection reflect the state of our world in late 2020, and they collectively represent several different perspectives, upon what has been happening and what is going to happen to our lives, planet, socio-economical relationships, and interpretations of meanings of life. The questions explored by the authors from the nature of viruses, the history of RNA research, human-animal relations, and implications of COVID-19 for clinical knowledge, public health policy, and structural inequality, and mental health. They also deal with broader questions connected to the nexus of health life sciences with freedom, identity, uncertainty, and scepticism, to name a few, and the relationship between scientific and health knowledge and different forms of media. Each paper of the Topical Collection "Seeing Clearly Through COVID-19" may be understood as a piece of a puzzle, and only by viewing the entire group of papers can we gain a more complete image of the complexity of the situation. In this way, indeed, it is possible to grasp the variety of problems, solutions, expectations, worries that in different countries in the world have faced. This collection of short essays offers a snapshot of how our knowledges 
of the pandemic have already changed in a short period of time. As an archive of reflections, they may serve and guide further scholarship, analysis, and policy.

\section{Declarations}

Conflict of interest The authors declare that they have no conflict of interest.

Publisher's Note Springer Nature remains neutral with regard to jurisdictional claims in published maps and institutional affiliations. 